\title{
Analysis of Theoretical and Experimental Results on the Effect of Injection Pressure in the Pump Section on the Working Processes and Characteristics of a Piston Hybrid Power Machine with a Slotted Seal of a Stepped Type
}

\author{
Victor E. Shcherba, \\ Victor V. Shalai, Aleksandr V. Grigor'yev, \\ Aleksey M. Bazhenov and Aleksey Yu. Kondyurin* \\ Omsk State Technical University \\ 11 Mira Str., Omsk, 644050, Russia
}

Received 13.02.2018, received in revised form 03.03.2018, accepted 06.05.2018

The paper presents the results of experimental and theoretical studies of a piston hybrid power machine with a slotted seal of a stepped type. A mathematical model of the working processes of the machine running in the pump section is presented. The analysis of the influence of the discharge pressure in the pump section on the characteristics of a piston hybrid power machine with a slotted seal of a stepped type that allowed to prove the efficiency of using a staged slit seal in the PGPM in comparison with a smooth seal, and also to determine the optimum operation of the PGPM at a discharge pump pressure in the interval 3-4 bar.

Keywords: pump, compressor, hybrid machine.

Citation: Shcherba V.E., Shalai V.V., Grigor'yev A.V., Bazhenov A.M., Kondyurin A.Yu. Analysis of theoretical and experimental results on the effect of injection pressure in the pump section on the working processes and characteristics of a piston hybrid power machine with a slotted seal of a stepped type, J. Sib. Fed. Univ. Eng. technol., 2018, 11(5), $591-603$. DOI: $10.17516 / 1999-494 X-0058$.

(c) Siberian Federal University. All rights reserved

* Corresponding author E-mail address: scherba_v_e@list.ru 


\title{
Анализ теоретических и экспериментальных результатов по влиянию давления нагнетания в насосной секции на рабочие процессы и характеристики поршневой гибридной энергетической машины с щелевым уплотнением ступенчатого вида
}

\author{
В.Е. Щерба, В.В. Шалай, \\ А.В. Григорьев, А.М. Баженов, А.Ю. Кондюрин \\ Омский государственный технический университет \\ Россия, 644050, Омск, ул. Мира, 11
}

В работе приведены результаты экспериментальных и теоретических исследований поршневой гибридной энергетической машины с щелевым уплотнением ступенчатого вида. Представлена математическая модель рабочих проиессов, протекаюшчх в насосной секции машины. Проведен анализ влияния давления нагнетания в насосной секции на характеристики поршневой гибридной энергетической машины с щелевым уплотнением ступенчатого вида, который позволил доказать эффективность использования в ПГЭМ ступенчатого шелевого уплотнения в сравнении с гладким уплотнением, а также определить оптимальную работу ПГЭМ при давлении нагнетания насосной секциии в интервале 3-4 бар.

Ключевые слова: насос, компрессор, гибридная машина.

\section{Введение}

При исследовании новых конструкций объемных гидромашин одной из главных задач является оценка внешних характеристик данных машин при различных давлениях в линии нагнетания [1].

В поршневой гибридной энергетической машине (ПГЭМ) работа насосной секции принципиально не отличается от работы поршневого насоса, и исследование влияния давления нагнетания в насосной секции на ее характеристики также одно из важнейших исследований [2].

Одной из перспективных конструкций поршневых гибридных энергетических машин является ПГЭМ с поршневым уплотнением ступенчатого вида [3].

Следует отметить, что работа ступенчатого уплотнения в данной машине основана на увеличении гидравлического сопротивления щели при движении поршня от верхней мертвой точки (BMT) к нижней (НMT) за счёт увеличения длины $1_{1}$ щелевого уплотнения с малым значением радиального зазора $\delta_{1}$ и уменьшения длины $l_{2}$ с большим значением радиального зазора $\delta_{2}$, и уменьшения гидравлического сопротивления при движении от НMT к ВМТ за счет увеличения $1_{2}$ и уменьшения $1_{1}$ [4]. Применение данного уплотнения позволяет улучшить энергетические и экономические характеристики ПГЭМ, а в частности, снизить теплонапряженность цилиндро-поршневой группы машины и ликвидировать мертвое пространство компрессорной секции.

В связи с тем, что давление нагнетания насосной секции оказывает существенное влияние на рабочие процессы ПГЭМ с гладким щелевым уплотнением [5], настоящая работа посвящена 
исследованию влияния давления нагнетания в насосной секции на рабочие процессы и внешние характеристики ПГЭМ с поршневым уплотнением ступенчатого вида.

\section{Экспериментальное исследование}

Целью натурных экспериментов в настоящей работе является подтверждение адекватности математической модели исследуемого технического объекта, которое производится сравнением данных, добытых расчетным и экспериментальным путем, а также получение эмпирических знаний о температурных режимах работы ПГЭМ.

Экспериментальный образец представляет собой поршневую машину с крейцкопфным направлением дифференциального поршня (рис. 1), который делит общий цилиндр на две ча-

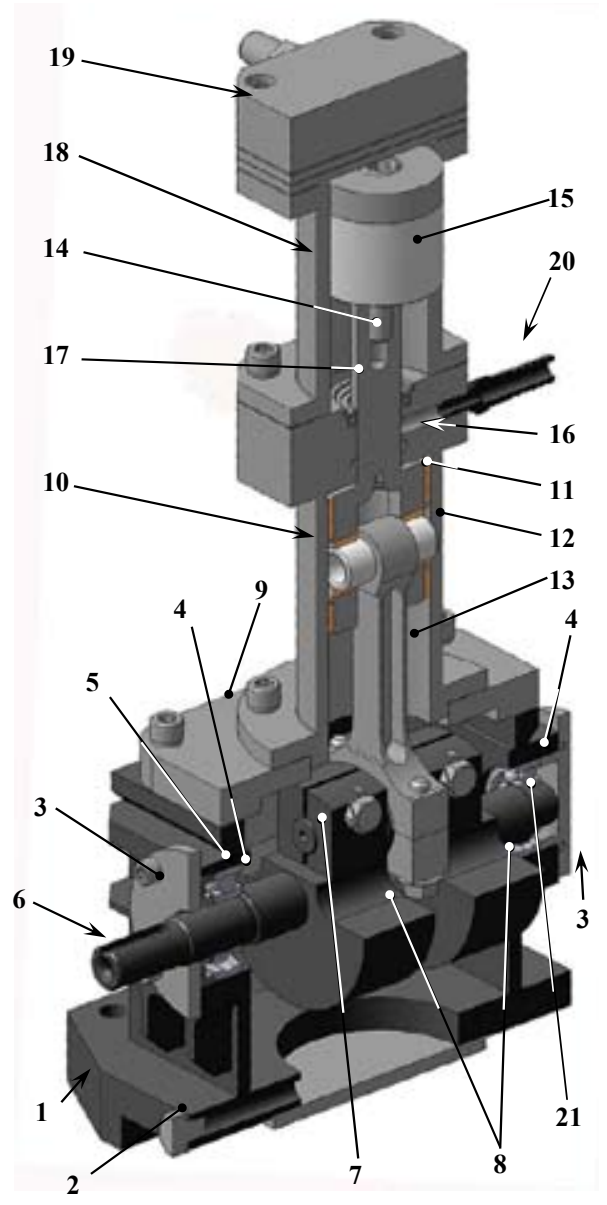

Рис. 1. 3D-изображение компоновки экспериментального образца поршневой гибридной машины: 1. Картер. 2. Заглушка. 3. Крышка подшипника. 4. Подшипник. 5. Сальник. 6. Приводной вал. 7. Кривошип. 8. Противовес. 9. Крышка картера. 10. Направляющая крейцкопфа. 11. Крейцкопф. 12. Палец. 13. Шатун. 14. Шток. 15. Поршень. 16. Сальник. 17. Уплотнение. 18. Цилиндр. 19. Клапанная коробка. 20. Дренаж. 21. Задняя опора

Fig. 1. 3D image of the layout of the experimental sample of a hybrid piston engine: 1. Carter. 2. Stopper. 3. Bearing cover. 4. Bearing. 5. Omentum. 6. Drive shaft. 7. Crank. 8. Counterweight. 9. Crankcase cover. 10. Crosshead guide. 11. Crosshead. 12. The finger. 13. Connecting rod. 14. Stock. 15. The piston. 16. Omentum. 17. Sealing. 18. Cylinder. 19. Valve box. 20. Drainage. 21. Rear support 
сти - надпоршневую газовую, или компрессорную, полость и подпоршневую (штоковую) жидкостную, или насосную [6].

С учетом имеющегося опыта экспериментального исследования поршневых гибридных машин [7] для построения конструкции экспериментального образца были выбраны следующие основные геометрические характеристики: диаметр цилиндра 0,05 м; ход поршня 0,05 м; общая рабочая длина цилиндра 0,1 м; длина верхней уплотняющей части 0,055 м; длина нижней уплотняющей части 0,045 м; зазор между поршнем и цилиндром в верхней части 14 мкм; зазор между поршнем и цилиндром в нижней части 61,5 мкм; длина поршня 0,049 м.

При проведении экспериментальных исследований в качестве независимого параметра выбиралось давление нагнетания в насосной секции $-p_{н н}$.

Также при проведении экспериментальных исследований принималось, что номинальное давление всасывания в насосной и компрессорной секциях одинаково $\left(p_{\text {вск }}=p_{\text {всн }}\right)$ и равно 1 бар.

В качестве функций отклика взяты:

1. Количество жидкости, выносимой в линию нагнетания компрессорной секции в единицу времени (л/мин).

2. Параметры теплонапряженности цилиндропоршневой группы.

2.1. Температура поверхности клапанной плиты компрессорной секции $\left(T_{\kappa p}\right)$.

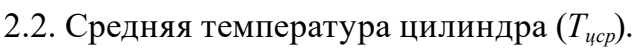

2.3. Средняя температура цилиндропоршневой группы $\left(\bar{T}_{c m}\right)$.

3. Температура всасываемого газа $\left(T_{\text {вск }}\right)$.

4. Коэффициент подачи в компрессорной секции $(\lambda)$.

5. Объемный к.п.д. насосной секции $\left(\eta_{\text {об) }}\right.$.

Для оценки эффективности работы щелевого уплотнения ступенчатого вида были изготовлены два цилиндра:

1. Цилиндр с щелевым уплотнением ступенчатого вида, имеющий следующие параметры: $\ell_{1}=0,055 \mathrm{~m} ; \delta_{1}=14$ мкм; $\ell_{2}=0,045 \mathrm{~m} ; \delta_{2}=61,5$ мкм.

2. Цилиндр с гладким щелевым уплотнением $\delta_{l}=\delta_{2}=6$ мкм.

Необходимо отметить, что поршень, а также остальные детали ПГЭМ оставались теми же. Длина поршня $\ell_{P}=0,049$ м. При планировании эксперимента применяли классический план с дробными репликами. Использовался следующий диапазон изменения давление нагнетания в насосной секции 4 бар $\leq p_{\text {ни }} \leq 11$ бар. Экспериментальные исследования проводились при $p_{н к}=5$ бар и $n_{о б}=280$ об/мин.

В качестве сжимаемого газа выступал воздух, в качестве рабочей жидкости - гидравлическое масло Роснефть МГЕ-46В.

Проведем последовательное рассмотрение влияния на рабочие процессы и эксплуатационные характеристики давления нагнетания в насосной секции.

С увеличением давления нагнетания в насосной секции увеличивается количество жидкости, поступающей в компрессорную секцию, и, соответственно, количество жидкости, поступающей в линию нагнетания компрессорной секции $\Delta V_{w}$. Для ПГЭМ с гладким щелевым уплотнением величина $\Delta V_{w}$ практически не меняется от $p_{\text {нн }}$ истается в десятки раз меньше, чем для ПГЭМ с щелевым ступенчатым уплотнением. Таким образом, по результатам эксперимента можно считать, что жидкость из насосной секции в компрессор- 
ную при использовании ступенчатого уплотнения не поступает на всем диапазоне изменения величины $p_{н н}$.

Увеличение количества охлаждающей жидкости приводит к улучшению охлаждения деталей цилиндро-поршневой группы. Так, увеличение давления нагнетания $p_{н н}$ с до 11 бар приводит к снижению температуры поверхности клапанной плиты с 326,3 до 323,5 К, т.е. почти на 3 К, аналогично уменьшается температура поверхности стенок цилиндра и средняя температура поверхности рабочей камеры компрессорной секции $\bar{T}_{c m}$, значение которой определялось следующим образом:

$$
\bar{T}_{c m}=\frac{T_{\kappa p} F_{\kappa p}+\bar{T}_{u} F_{u}+F_{p} \cdot T_{w \mu}}{F_{\kappa p}+F_{u}+F_{p}},
$$

где $F_{\kappa p}, F_{u}, F_{p}$ - площадь поверхности клапанной плиты, цилиндра и поршня; $T_{\kappa p}, \bar{T}_{u}, T_{w н}-$ температура поверхности клапанной плиты, цилиндра и жидкости на нагнетании насоса.

В первом приближении принимаем, что температура жидкости на поверхности днища поршня равна температуре жидкости на нагнетании насосной секции. Увеличение $p_{н н}$ приводит к уменьшению температуры газа на всасывании компрессорной секции с 309,2 до 307,8 К вследствие улучшения охлаждения цилиндропоршневой группы (рис. 2).

Для ПГЭМ с гладким щелевым уплотнением увеличение $p_{н н}$ не оказывает влияния на температуру поверхности клапанной плиты. Ее величина выше почти на $6 \mathrm{~K}$, чем $T_{\kappa p}$ с гладким щелевым уплотнением. Величина $\bar{T}_{u}$ также остается практически постоянной и равной 317 К. Температура всасываемого воздуха остается также неизменной и равной 309 К, что на 1,2 К выше, чем у ПГЭМ со ступенчатым щелевым уплотнением.

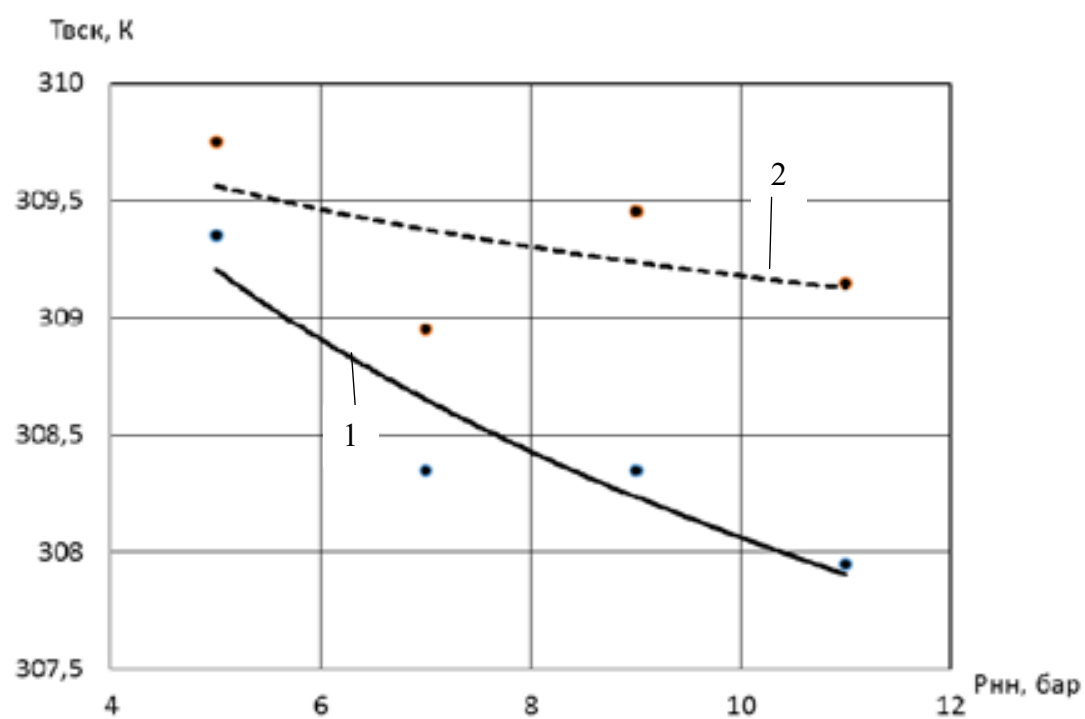

Рис. 2. Экспериментальная зависимость температуры всасываемого газа от давления нагнетания насосной секции: 1 - ПГЭМ с щелевым уплотнением ступенчатого вида; 2 - ПГЭМ с гладким щелевым уплотнением

Fig. 2. Experimental dependence of the intake gas temperature on the discharge pressure of the pump section: 1 - PGEM with a slotted seal of a stepped type; 2 - PGEM with a smooth slit seal 


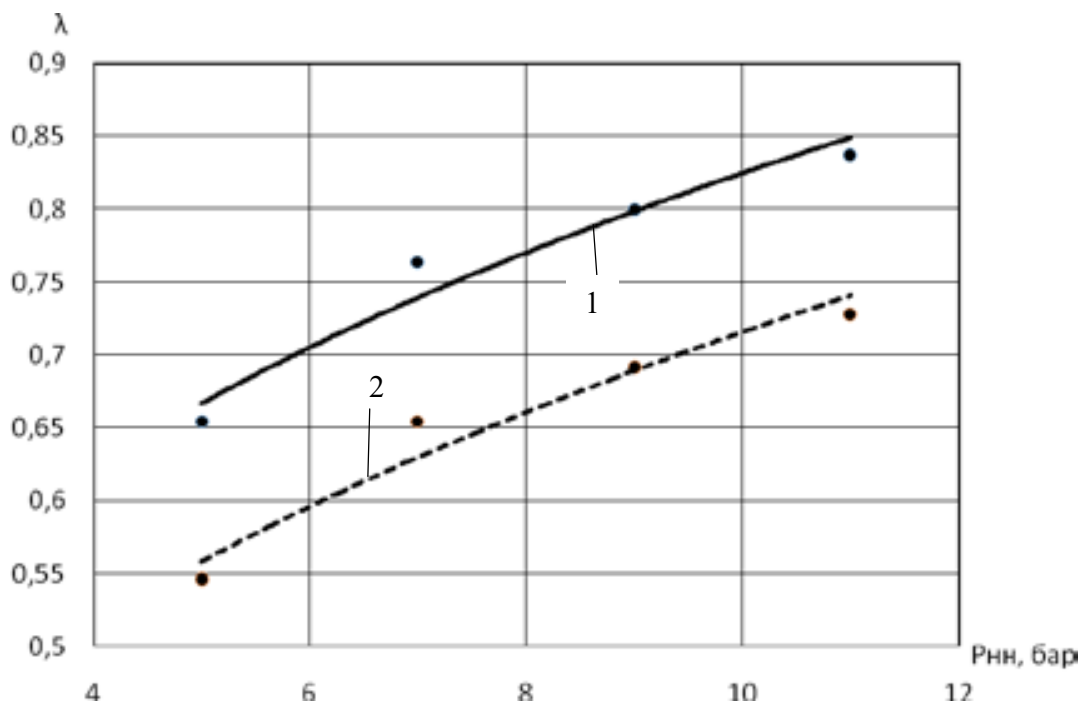

Рис. 3. Экспериментальная зависимость коэффициента подачи компрессорной секции от давления нагнетания насосной секции: 1 - ПГЭМ с щелевым уплотнением ступенчатого вида; 2 - ПГЭМ с гладким щелевым уплотнением

Fig. 3. Experimental dependence of the feed rate of the compressor section on the discharge pressure of the pump section: 1 - PGEM with a slotted seal of a stepped type; 2 - PGEM with a smooth slit seal

Увеличение жидкости в сжимаемом газе при увеличении $p_{н н}$ приводит к уменьшению мертвого пространства, что увеличивает значение объемного коэффициента $\lambda_{\text {o }}$. Уменьшение температуры всасываемого газа приводит к увеличению температурного коэффициента $\lambda_{T}$. Увеличение $\lambda_{o}$ и $\lambda_{T}$ приводит к увеличению $\lambda$ с 0,655 при $p_{н \mu}=5$ бар до 0,837 до $p_{\mu н}=11$ бар (рис. 3 ). Для ПГЭМ с гладким щелевым уплотнением с увеличением $p_{\text {нн }}$ количество жидкости в рабочей полости компрессорной секции увеличивается, что приводит к уменьшению мертвого пространства и увеличению $\lambda$ с 0,545 при $p_{н н}=5$ бар до 0,727 при $p_{н н}=11$ бар. Таким образом, во всем диапазоне изменения $p_{н н}$ у ПГЭМ со ступенчатым щелевым уплотнением величина $\lambda$ более чем на 10 \% больше, чем у ПГЭМ с гладким щелевым уплотнением.

С увеличением $p_{н н}$ величина $\eta_{о б}$ уменьшается как для ступенчатого уплотнения, так и для

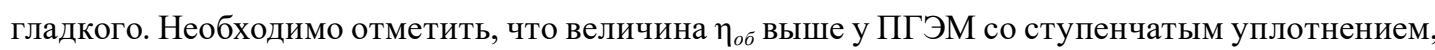
чем с гладким, несмотря на то, что количество жидкости, выносимой в линию нагнетания компрессорной секции, в первом случае выше, чем во втором.

\section{Теоретическое исследование}

Помимо экспериментальных исследований по влиянию давления нагнетания в насосной секции на работу исследуемой ПГЭМ, также посредством математической модели рабочих процессов ПГЭМ были проведены теоретические исследования, которые позволили существенно сократить затраты на проведение каждого опыта.

Математическая модель рабочих процессов в компрессорной секции включает в себя уравнение первого закона термодинамики тела переменной массы, уравнение сохранения массы, уравнение движения, уравнение состояния газа [8].

$$
-596-
$$


Математическая модель рабочих процессов насосной секции базируется на двух системах уравнений, которые в конечном виде записываются таким образом:

- система уравнений, описывающая изменение термодинамических параметров в процессах сжатия и расширения в насосной полости:

$$
\left\{\begin{array}{l}
V_{w}=V_{M w}+\frac{V h}{2}\left[(1-\cos \varphi)+\frac{\lambda}{4}(1-\cos 2 \varphi)\right] \\
d M_{w}=\sum_{i=1}^{n_{1}} d M_{n i w}-\sum_{i=1}^{n_{2}} d M_{0 i w} \\
P_{w}=P_{w_{B C}}+E_{w}\left(\ln \frac{V_{w_{B C}}}{V_{w}}+\ln \frac{M_{w}}{M_{w_{B C}}}\right) \\
T_{w}=\text { const }
\end{array}\right.
$$

где $V_{w}, M_{w}, P_{w}, T_{w}$ - текущие значения объема, массы, давления и температуры жидкости в рабочей полости насосной секции соответственно; $d M_{n i w}, d M_{0 i w}-$ элементарные массы присоединяемой и отделяемой жидкости; $d M_{w}$ - элементарное изменение массы жидкости; $V_{w \in c}, P_{\text {wsc }}, M_{\text {wвc }}-$ объем, давление и масса жидкости в конце процесса всасывания; $E_{w}$ - модуль упругости жидкости; $V h, V_{M}$ - величина рабочего и мертвого объема в рабочей полости насосной секции; $\varphi$ - угол поворота коленчатого вала;

- система уравнений, описывающая процессы нагнетания и всасывания в насосной полости:

$$
\left\{\begin{array}{l}
p_{w}=p_{w_{H}}+\rho_{w} g\left(S_{M w}+S_{w}\right) \cos \alpha+\rho_{w} \frac{w_{2}^{2}-w_{1}^{2}}{2}+\Delta h_{T 1-2}+\Delta h_{1-2} \\
m_{n p w} \frac{d^{2} h_{w}}{d \tau^{2}}=\sum_{i=1}^{n_{3}} F_{i w}
\end{array}\right.
$$

где $p_{w н}$ - давление жидкости в линии нагнетания; $S_{w}$ - текущее значение перемещения поршня; $w_{1}$ - скорость жидкости в сечении I-I, совпадающем с днищем поршня; $w_{2}$ - скорость жидкости в сечении II-II, расположенном за нагнетательным клапаном; $\Delta h_{T 1-2}-$ потери напора, обусловленные совершением технической работы на пути между выбранными сечениями; $\Delta h_{1-2}-$ потери напора на трение (по длине и местные сопротивления); $S_{M w}-$ линейный мертвый объем; $\alpha$ - угол между вертикалью и осью цилиндра; $\rho_{w}$ - плотность жидкости; g - ускорение свободного падения; $m_{n p w}-$ приведенная масса запорного органа самодействующего клапана насосной секции; $h_{w}$ - текущая высота подъема запорного органа самодействующего клапана насосной секции; $\Sigma F_{I W}$ - сумма сил, действующих на запорный орган самодействующего клапана; $\tau-$ время.

Моделирование процессов, протекающих в ступенчатом щелевом уплотнении, основывается на уравнении расхода жидкости через гладкое концентричное щелевое уплотнение у поршневого насоса и уравнении неразрывности жидкости в широкой и узкой частях уплотнения.

При проведении анализа влияния давления нагнетания в насосной секции примем, что давление нагнетания в компрессорной секции равно 1,0 МПа. В общем случае, вследствие

$$
-597-
$$


особенностей индикаторной диаграммы в насосной секции (близка к прямоугольнику) давление в насосной секции оказывает более значимое влияние на количество перетекаемой жидкости через поршневое уплотнение за цикл, чем давление нагнетания в компрессорной секции $[9,10]$.

С увеличением давления нагнетания в насосной секции увеличивается количество охлаждающей жидкости в рабочей полости компрессорной секции. Увеличение количества охлаждающей жидкости (или ее появление в начале процесса нагнетания) деформирует кривую процесса нагнетания, увеличивая потери давления в процессе нагнетания. Процессы сжатия, обратного расширения и всасывания изменяются незначительно и потери давления в процессе всасывания остаются практически постоянными.

Аналогично изменению относительных потерь давления в процессах всасывания и нагнетания в компрессорной секции ведут себя относительные потери работы в процессах всасывания и нагнетания. Индикаторные диаграммы в насосной секции при разных давлениях нагнетания представлены на рис. 4. Ступенчатое изменение давления в процессе сжатия обусловлено поздним закрытием всасывающего клапана в насосной секции (кривая 1 рис. 5). Нетрудно увидеть, что изменение давления нагнетания в насосной секции оставляет потери давления в процессах нагнетания и всасывания постоянными. Уменьшение относительных потерь работы обусловлено увеличением работы цикла в насосной секции $A_{\psi w}$.

С увеличением давления нагнетания в насосной секции высота слоя жидкости над поршнем увеличивается (ордината точки А рис. 6), процесс выталкивания жидкости увеличивается (кривая ВС). При давлении нагнетания в насосной секции $p_{н t} \leq 2,5$ бар процесс выталкивания жидкости ВС вырождается в точку.

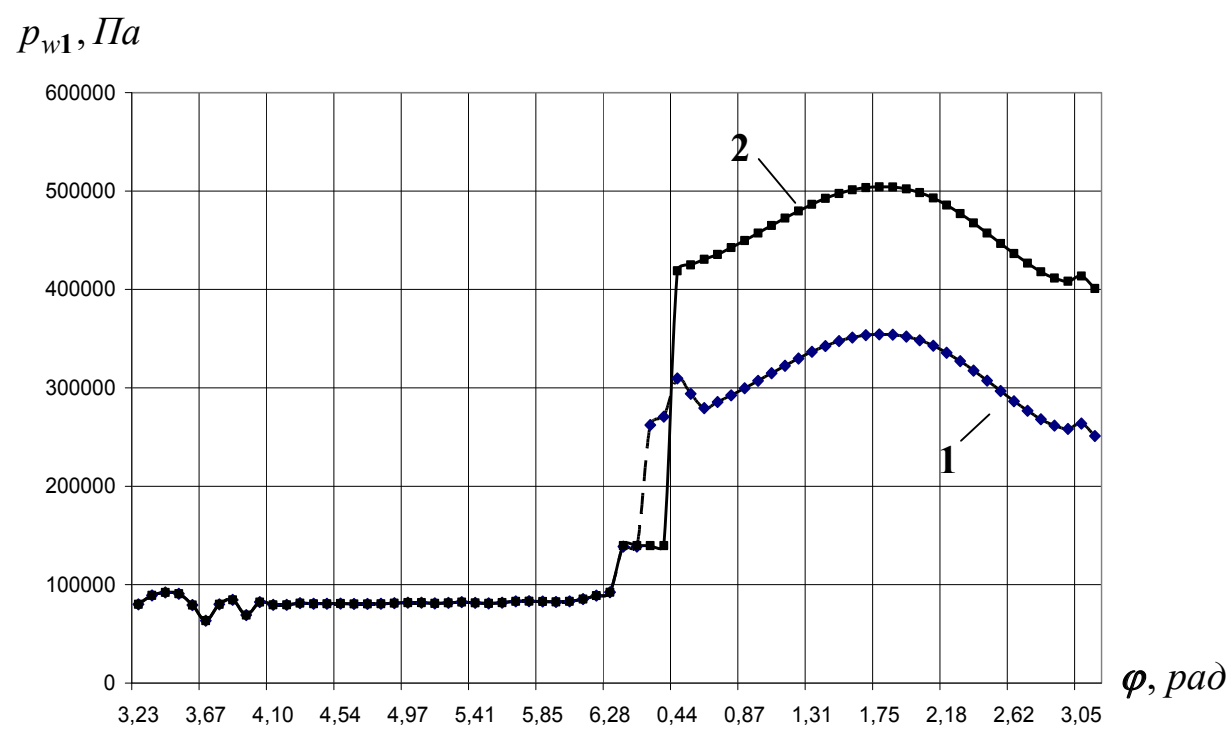

Рис. 4. Теоретические индикаторные диаграммы в насосной секции при разных значениях давления нагнетания: 1 - 250000 Па; 2 - 400000 Па

Fig. 4. Theoretical indicator diagrams in the pump section at different discharge pressures: $1-250000 \mathrm{~Pa}$; $2-400000 \mathrm{~Pa}$ 


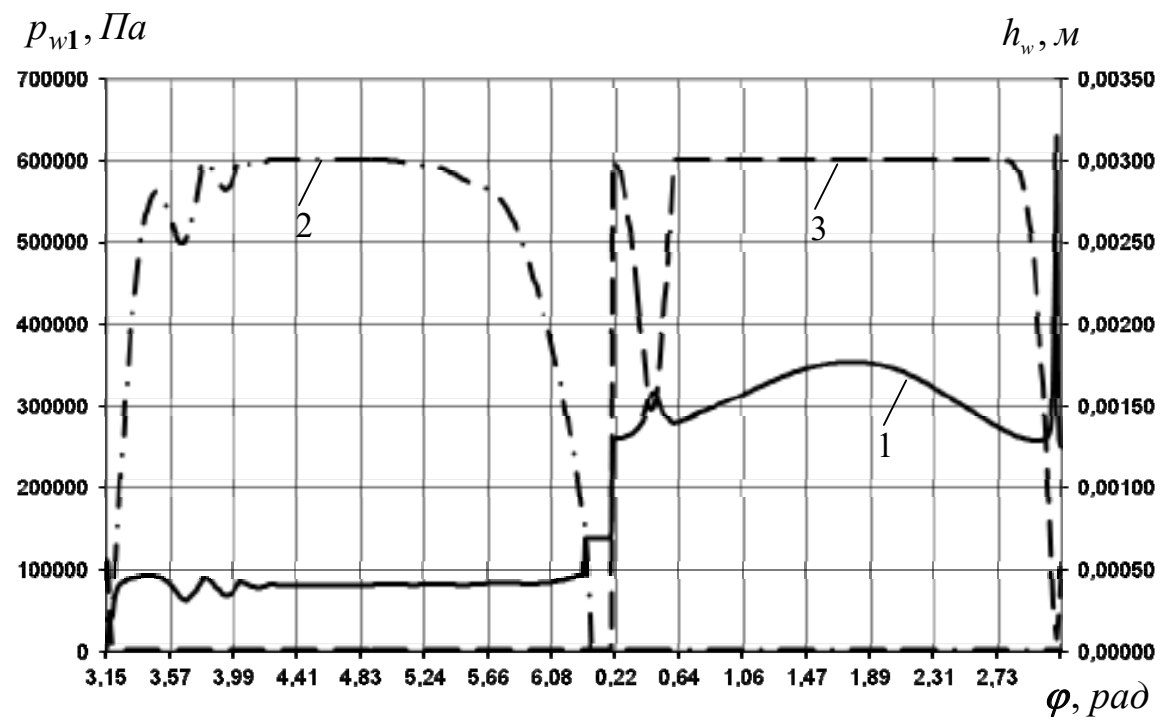

Рис. 5. Теоретическая индикаторная диаграмма в насосной полости (1) и диаграммы движения запорных элементов клапанов: 2 - всасывающий клапан; 3 - нагнетательный клапан

Fig. 5. Theoretical indicator diagram in the pump cavity (1) and diagrams of movement of the valve shut-off elements: 2 - suction valve; 3 - discharge valve

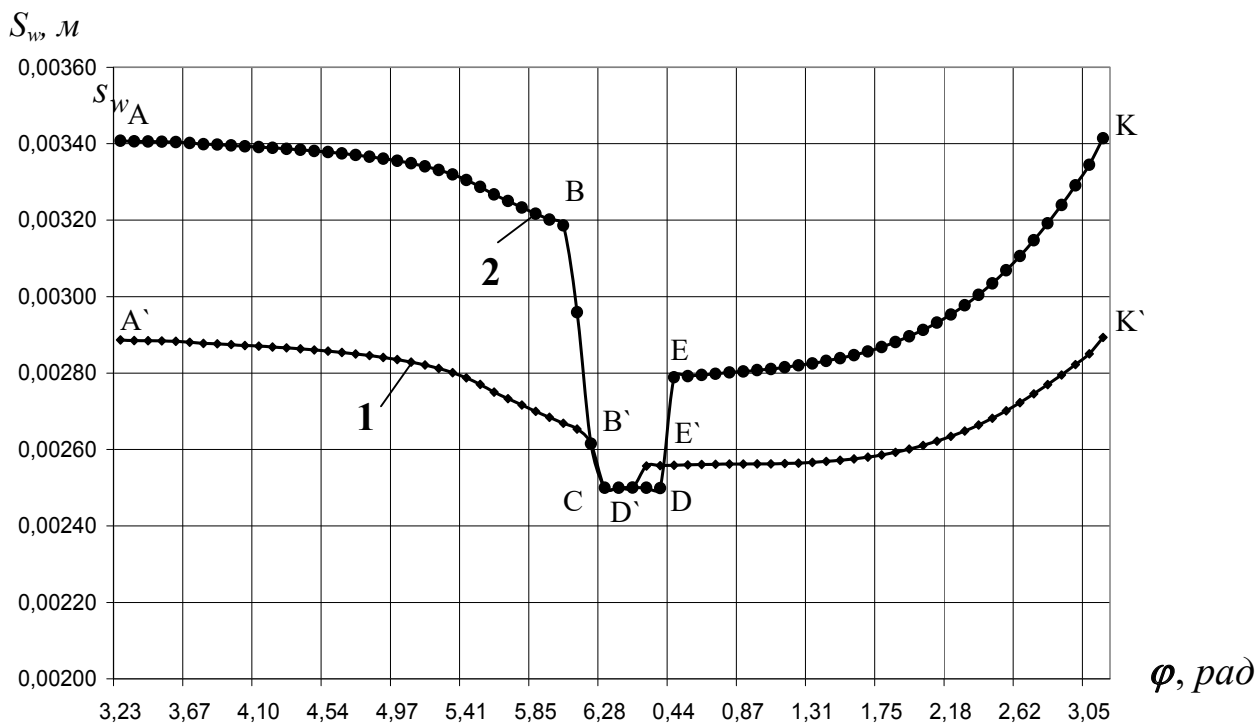

Рис. 6. Экспериментальная зависимость высоты слоя жидкости над поршнем от угла поворота коленчатого вала при давлении нагнетания в насосной секции: 1 - 250000 Па; 2 - 400000 Па

Fig. 6. Experimental dependence of the height of the liquid layer above the piston on the angle of rotation of the crankshaft at the injection pressure in the pump section: $1-250000 \mathrm{~Pa} ; 2-400000 \mathrm{~Pa}$ 
Представленные результаты на рис. 7 подтверждают сделанный вывод. Интенсивный рост $\bar{G}_{w \kappa}$ начинается при давлении нагнетания более 3,5 бар. Величина $G_{\text {отн }}$ приближается к 1 при давлении нагнетания 2,5 бар и меньше. Относительная высота слоя жидкости за цикл $\bar{S}_{w_{c p}}$ интенсивно увеличивается в диапазоне изменения давлений нагнетания в насосной секции от 2 до 3 бар. Дальнейшее увеличение давления в насосной секции не приводит к значительному увеличению $\bar{S}_{w_{c p}}$. Это обусловлено тем, что избыточная жидкость вытесняется через нагнетательный клапан в компрессорной полости.

При уменьшении давления нагнетания в насосной секции меньше 3 бар жидкость к концу процесса нагнетания не заполняет полностью мертвое пространство. Так, при давлении нагнетания 2,5 бар 75,2 \% мертвого пространства занято газом, а при 2,0 бар $100 \%$ мертвого пространства занято газом. Увеличение мертвого пространства, занятого газом, приводит к уменьшению объемного коэффициента от 1 при $p_{н н}=3$ бар до 0,7438 при $p_{н н}=2$ бар. Величина коэффициента дросселирования $\lambda_{p}$ с увеличением $p_{н н}$ незначительно уменьшается, что обусловлено увеличением потерь давления в процессе всасывания вследствие увеличения процесса всасывания при уменьшении мертвого пространства, занятого газом. С увеличением $p_{н н}$ наблюдается также незначительное уменьшение $\lambda_{T}$ (рис. 8).

Вследствие значительного увеличения объемного коэффициента $\lambda_{0}$ и незначительного уменьшения коэффициентов величина коэффициента подачи $\lambda$ с увеличением $p_{н н}$ увеличивается с 0,557 при $p_{н н}=2$ бар до 0,801 при $p_{н н}=4$ бар. Максимальные значения коэффициента по-

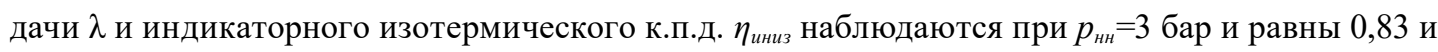
0,562 соответственно. Максимум индикаторного изотермического к.п.д. обусловлен, с одной стороны, увеличением производительности компрессорной секции при увеличении $p_{н н}$ (это увеличивает $\eta_{\text {ииз }}$ ), а с другой - увеличением потерь работы в процессе нагнетания (это ведет

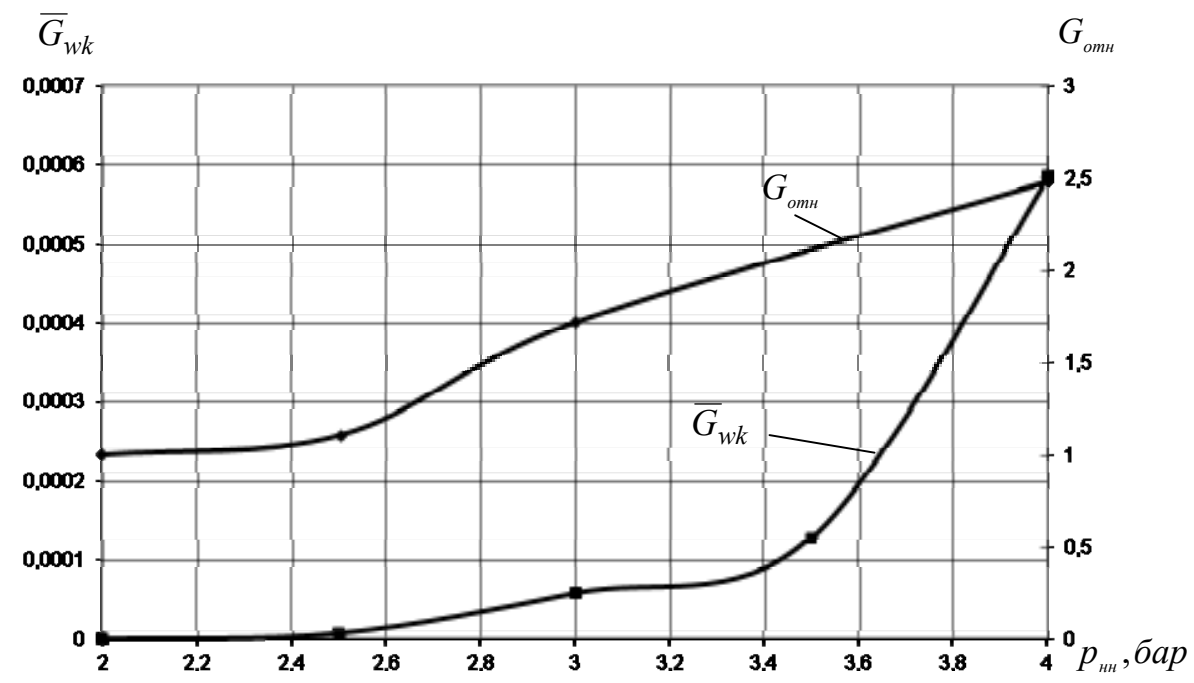

Рис. 7. Экспериментальная зависимость относительных величин $\bar{G}_{w k}$ и $G_{\text {оти }}$ от давления нагнетания в компрессорной секции

Fig. 7. Experimental dependence of the relative values and on the discharge pressure in the compressor section 


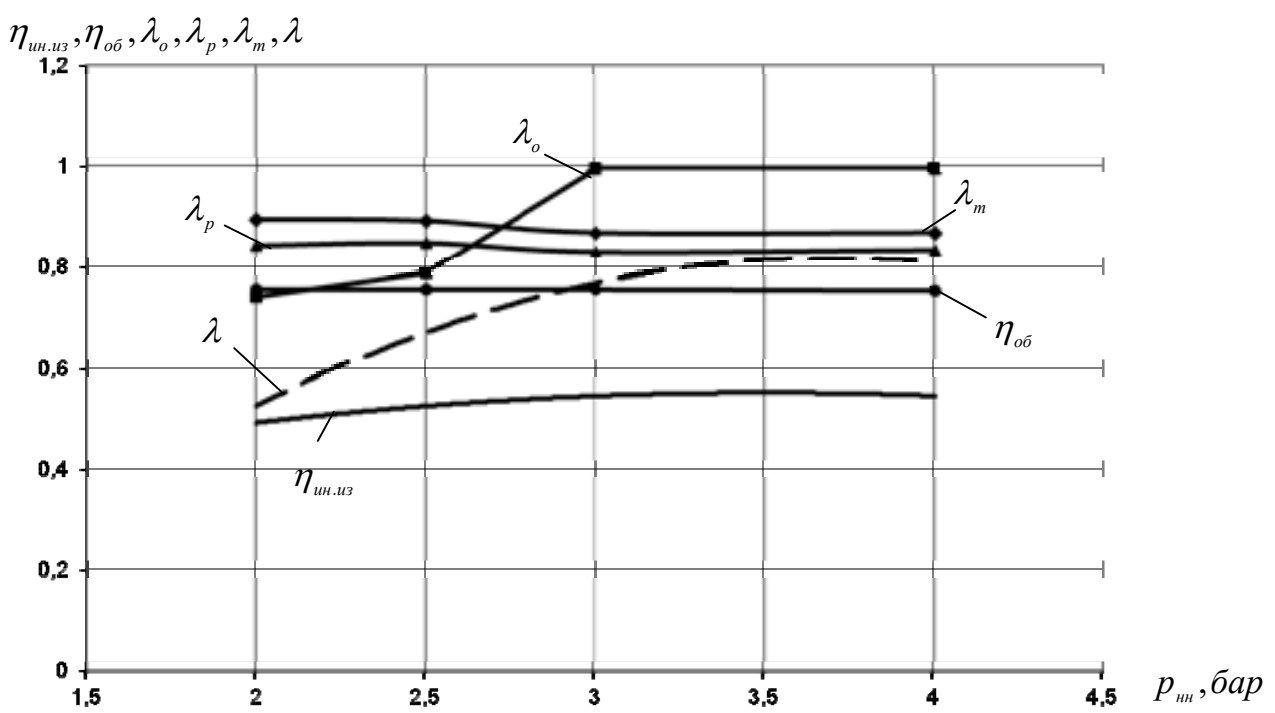

Рис. 8. Экспериментальная зависимость величин $\eta_{\text {иниз }}, \eta_{о \sigma}, \lambda_{o}, \lambda_{p}, \lambda_{m}, \lambda$ от давления нагнетания в насосной секции

Fig. 8. Experimental dependence of the quantities on the discharge pressure in the pump section

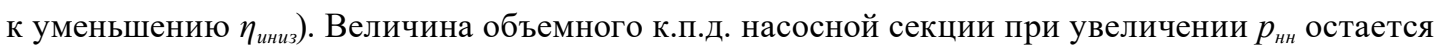
неизменной.

\section{Выводы}

Экспериментальные исследования доказали эффективность использования в ПГЭМ ступенчатого щелевого уплотнения в сравнении с гладким уплотнением. Применение ступенчатого уплотнения снижает температуру цилиндропоршневой группы и ликвидирует мертвое пространство компрессорной секции ПГЭМ.

Теоретически установлено, что диапазон давления нагнетания насосной секции для оптимальной работы ПГЭМ со ступенчатым щелевым уплотнением как для работы компрессорной, так и для насосной секции лежит в интервале 3-4 бар.

\section{Список литературы}

[1] Орлов Ю.М. Объемные гидравлические машины. Конструкиия, проектирование, расчет. М.: Машиностроение, 2006. 222 c. [Orlov Yu.M. Volumetric hydraulic machines. Construction, design, calculation. Moscow, Mechanical Engineering, 2006. 222 p. (in Russian)]

[2] Щерба В.Е., Болштянский А.П., Шалай В.В., Ходорева А.В. Насос-компрессоры. Paбочие процессы и основы проектирования. М.: Машиностроение, 2013. 367 с. [Shcherba V.E., Bolshtyansky A.P., Shalai V.V., Khodoreva A.V. Pump-compressors. Work processes and design basics. Moscow, Mechanical engineering, 2013. 367 p. (in Russian)]

[3] Щерба В.Е., Лысенко Е.А., Нестеренко Г.А., Григорьев А.В., Кондюрин А.Ю., Баженов А.М. Разработка и исследование поршневого уплотнения, выполненного в виде гладкой щели ступенчатого вида для поршневой гибридной энергетической машины объемно-

$$
-601-
$$


го действия, Химическое и нефтегазовое машиностроение, 2016, 4, 45-48. [Shcherba V.E., Lysenko E.A., Nesterenko G.A., Grigoriev A.V., Kondyurin A.Yu., Bazhenov A.M. Development and investigation of a piston seal, made in the form of a smooth slit of a step type for a piston hybrid power machine of volumetric action, Chemical and Oil and Gas Engineering, 2016, 4, 45-48. (in Russian)]

[4] Баженов А.М., Кондюрин А.Ю., Щерба В.Е. Расчет щелевого уплотнения, выполненного в виде гладкой щели ступенчатого вида поршневой гибридной энергетической машины объемного действия. Материаль ХХ Всероссийской научно-технической конференции студентов и аспирантов. М: НИУ МЭИ, 2016, 10-14. [Bazhenov A.M., Kondyurin A.Yu., Shcherba V.E. Calculation of a slot seal, made in the form of a smooth slit of a step-like type of a piston hybrid power machine of volumetric action. Materials of the XX All-Russian scientific and technical conference of students and post-graduate students. Moscow, 2016, 10-14. (in Russian)]

[5] Алтынцев М.П., Аверьянов Г.С., Суриков В.И., Виниченко В.С. Влияние отношения давления нагнетания к давлению всасывания компрессорной полости на характеристики поршневого насос-компрессора. Омский научный вестник, 2012, 1(107), 133-135. [Altyntsev M.P., Averyanov G.S., Surikov V.I., Vinichenko V.S. Influence of the ratio of discharge pressure to suction pressure of the compressor cavity on the characteristics of a reciprocating pump-compressor. Omsk Scientific Bulletin, 2012, 1 (107), 133-135. (in Russian)]

[6] Щерба В.Е., Шалай В.В., Лысенко Е.А., Нестеренко Г.А., Григорьев А.В., Баженов А.М. Разработка и создание испытательного стенда для исследований поршневой гибридной энергетической машины объемного действия. Материалы III Международной научно-технической конференции «Динамика и виброакустика машин». Самара: СНИУ им. академика С.П. Королева, 2016, 214-216. [Shcherba V.E., Shalay V.V., Lysenko E.A., Nesterenko G.A., Grigoriev A.V., Bazhenov A.M. Development and creation of a test bench for the research of a piston hybrid power machine of volumetric action. Materials of the III International Scientific and Technical Conference “Dynamics and Vibroacoustics of Machines". Samara, 2016, 214-216. (in Russian)]

[7] Кужбанов А.К., Щерба В.Е., Павлюченко Е.А., Нестеренко Г.А. Экспериментальные исследования поршневого насос-компрессора с газовым демпфером. Химическое и нефтегазовое машиностроение, 2015, 5, 28-32. [Kuzhbanov A.K., Shcherba V.E., Pavlyuchenko E.A., Nesterenko G.A. Experimental studies of a reciprocating pump-compressor with a gas damper. Chemical and Oil and Gas Engineering, 2015, 5, 28-32. (in Russian)]

[8] Пластинин П.И. Поршневые компрессоры. Т. 1: Теория и расчет. М.: Колос, 2000. 456 с. [Plastinin P.I. Reciprocating compressors. Vol. 1: Theory and calculation. Moscow, Kolos, 2000. 456 p. (in Russian)]

[9] Щерба В.Е., Болштянский А.П., Нестеренко Г.А., Кондюрин А.Ю. О соотношении массовых потоков жидкости и давлений нагнетания между насосной и компрессорной полостями в поршневой гибридной энергетической машине. Химическое и нефтегазовое машиностроение, 2016, 4, 35-38. [Shcherba V.E., Bolshtyansky A.P., Nesterenko G.A., Kondyurin A.Yu. On the ratio of mass flow of fluid and injection pressures between the pump and compressor cavities in a piston hybrid power machine. Chemical and Oil and Gas Engineering, 2016, 4, 35-38. (in Russian)]

[10] Баженов А.М., Щерба В.Е., Григорьев А.В., Кондюрин А.Ю., Парамонов А.М. Анализ влияния соотношения прямого и обратного потоков жидкости в щелевом уплотнении порш- 
невой гибридной энергетической машины на соотношение давлений нагнетания в насосной и компрессорной полостях. Омский научный вестник, 2016, 6(150), 45-49. [Bazhenov A.M., Shcherba V.E., Grigoriev A.V., Kondyurin A.Yu., Paramonov A.M. Analysis of the influence of the ratio of direct and reverse fluid flows in the slot seal of a piston hybrid power machine on the ratio of injection pressures in the pump and compressor cavities. Omsk Scientific Bulletin, 2016, 6(150), 45-49. (in Russian)] 